\title{
CORRECTION
}

\section{Correction to: A compression pipeline for one-stage object detection model}

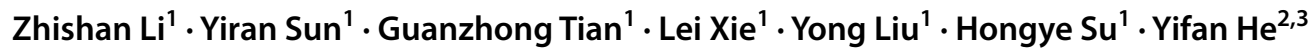

Published online: 10 February 2021

(c) Springer-Verlag GmbH Germany, part of Springer Nature 2021

\section{Correction to: Journal of Real-Time Image Processing https://doi.org/10.1007/s11554-020-01053-z}

In the original publication of the article, there is an error in the Table 5. The data in the Table 5 should be "- 55.99\%" but it has mistakenly published as "v55.99\%".

The correct table is given below.

The original article has been corrected.

Publisher's Note Springer Nature remains neutral with regard to jurisdictional claims in published maps and institutional affiliations.

The original article can be found online at https://doi.org/10.1007/ s11554-020-01053-z.

Lei Xie

leix@iipc.zju.edu.cn

1 State Key Laboratory of Industrial Control Technology and Institute of Cyber-systems and Control, Zhejiang

University, Hangzhou 310027, China

2 Reconova Technologies Co., Ltd, Xiamen 361008, China

3 Institute of Intelligence Science and Engineering, Shenzhen Polytechnic, Shenzhen 518055, China 
Table 5 Comparative experiment of pruning

\begin{tabular}{llcccc}
\hline Model & Method & Model size (MB) & Relative change (\%) & mAP (\%) & Relative change (\%) \\
\hline SSD & Baseline & 105.2 & 0.00 & 76.27 & 0.00 \\
& Network-Slimming [8] & 46.2 & -56.08 & 72.87 & -4.46 \\
& L1-Norm [21] & 46.2 & -56.08 & 74.35 & -2.52 \\
& FPGM [24] & 48.4 & -53.99 & 74.17 & -2.75 \\
& Rethinking-pruning [35] & 46.3 & -55.99 & 60.63 & -20.51 \\
& Our method & 46.3 & -55.99 & 75.18 & -1.43 \\
YoloV3 & Baseline & 142.1 & 0.00 & 78.03 & 0.00 \\
& Network-slimming [8] & 26.9 & -81.07 & 69.85 & -10.48 \\
& L1-Norm [21] & 26.2 & -81.56 & 73.78 & -5.45 \\
& FPGM [24] & 30.9 & -78.25 & 73.85 & -5.36 \\
& Rethinking-pruning [35] & 26.4 & -81.42 & 64.89 & -16.84 \\
& Our method & 26.4 & -81.42 & 75.44 & -3.32 \\
\hline
\end{tabular}

